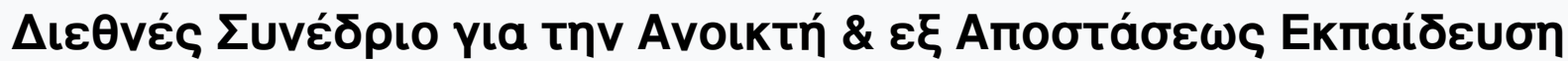

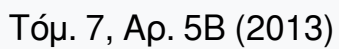

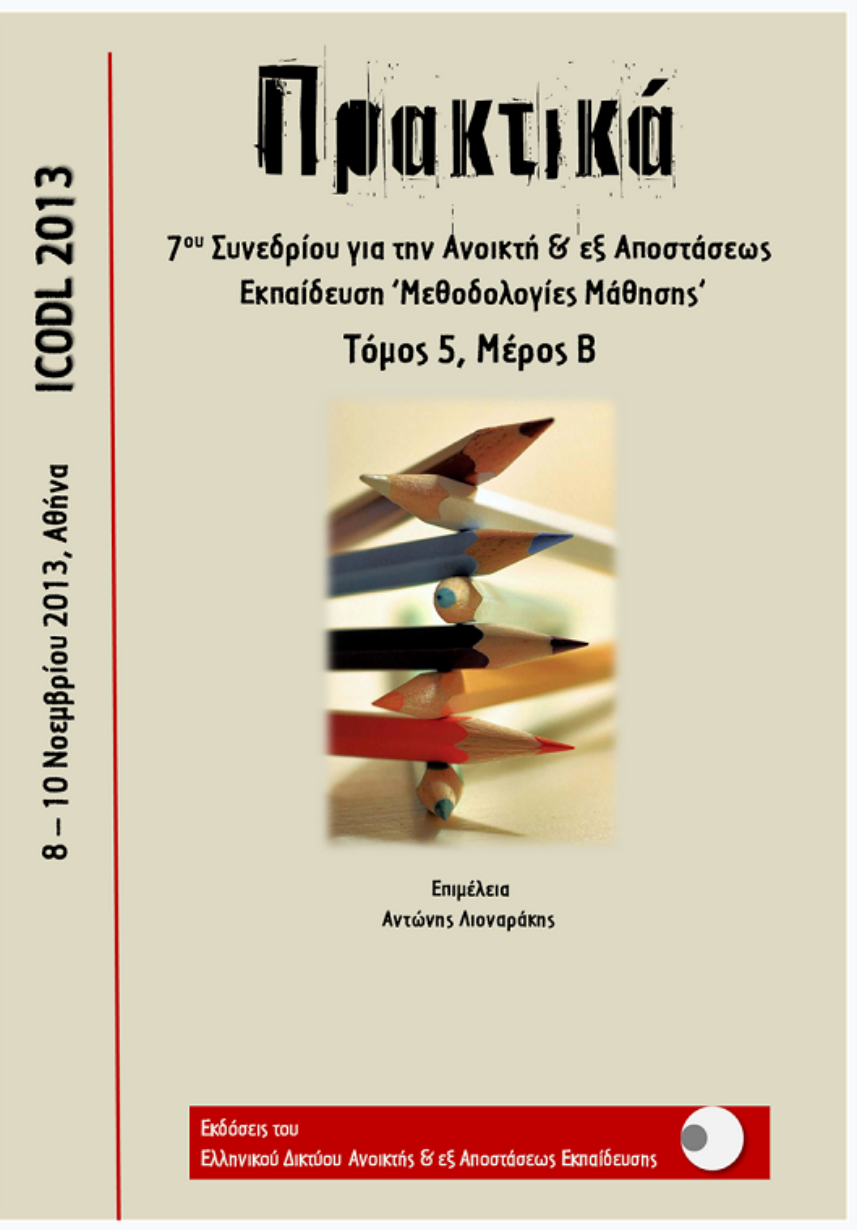

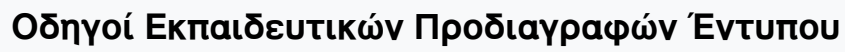

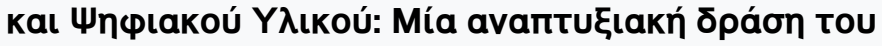

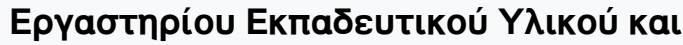

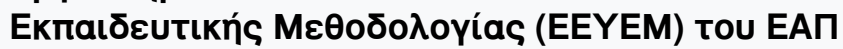

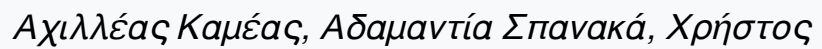

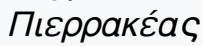

doi: $\underline{10.12681 / \text { icodl. } 556}$ 


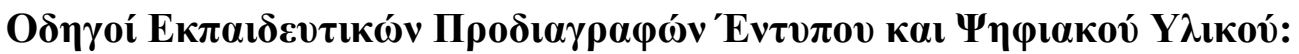

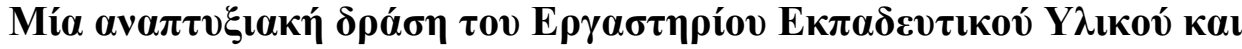

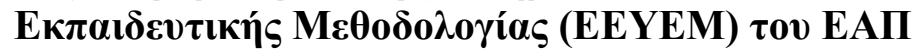

\section{Tutorials about pedagogic principles for print and digital distance learning materials: A developmental activity of e-CoMeT Laboratory of the Hellenic Open University}

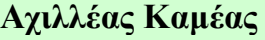

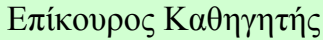

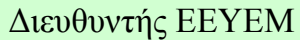 \\ ЕАП \\ kameas@eap.gr
}

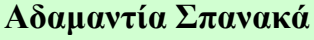 \\ ЕАП, К $\alpha \theta \eta \gamma \eta ́ \tau \rho ı-\Sigma v ́ \mu \beta o v \lambda o \varsigma$

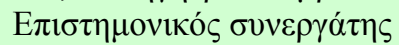

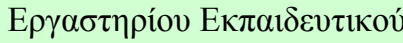

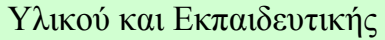

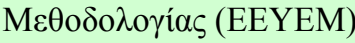 \\ madspa@otenet.gr
}

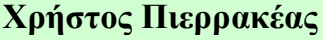

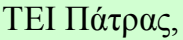

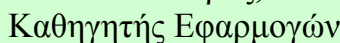 \\ Y $\pi \varepsilon v \dot{\theta v v o \varsigma ~ T o \mu \varepsilon ́ \alpha ~}$

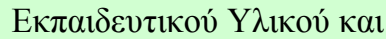

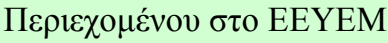 \\ ЕАП \\ pierrakeas@eap.gr
}

\begin{abstract}
In the Hellenic Open University, Educational Content Methodology and Technology Laboratory (e-CoMeT Lab, http://eeyem.eap.gr) among other tasks, developes methodologies for distance learning. In this context, e-CoMeT has developed a series of Tutorials or Toolkits about pedagogic principles for print and digital distance learning materials. These Tutorials outline a range of general techniques, activities and more specific ideas in order to offer a framework of guidelines to designers, creators and producers of distance learning material.
\end{abstract}

Keywords: Tutorials, pedagogic principles, print and digital distance learning materials, e-CoMeT Laboratory of Hellenic Open University

\section{Пері́ $\lambda \psi \eta$}

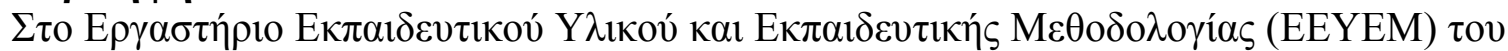

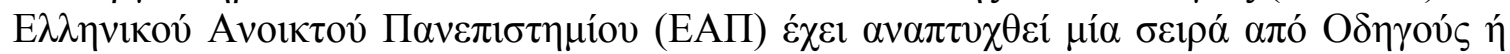

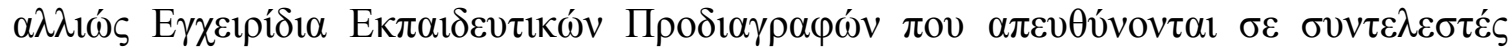

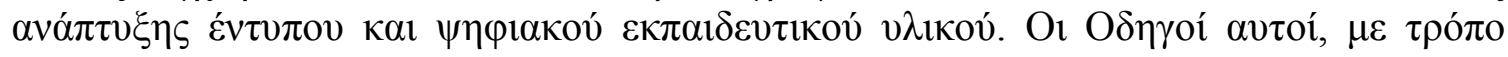

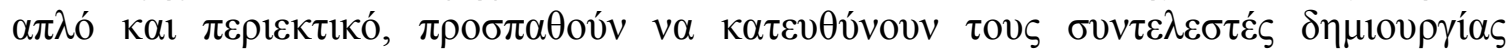

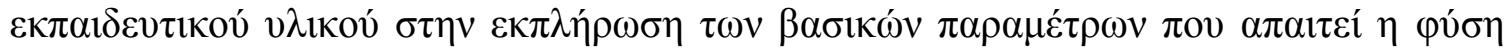

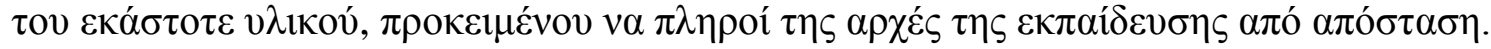

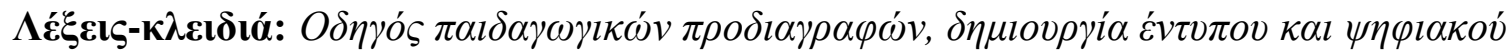

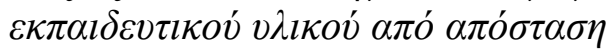

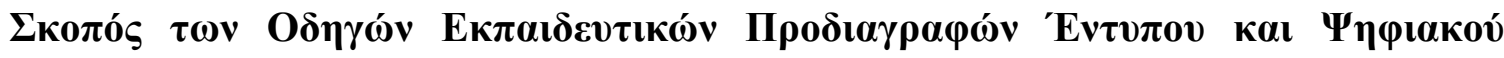

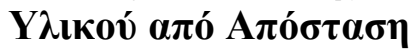

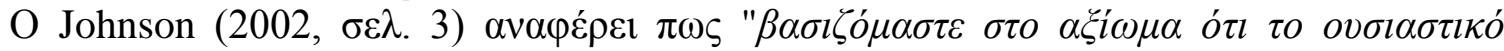

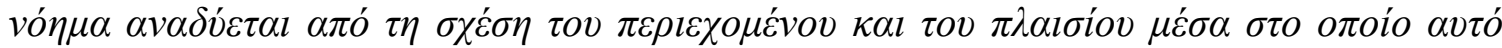

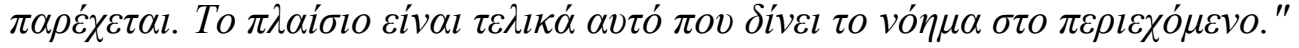




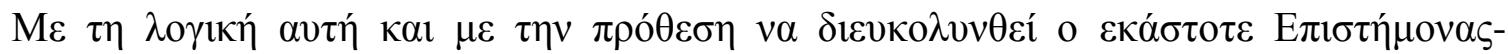

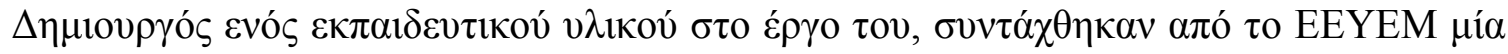

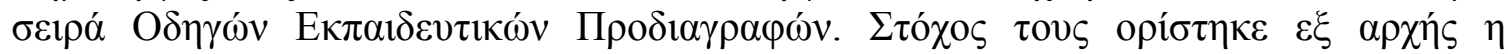

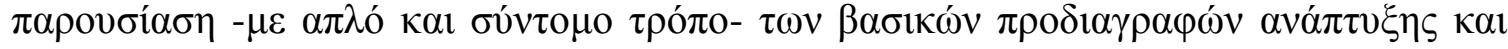

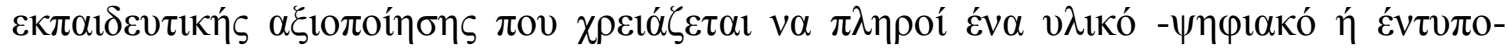

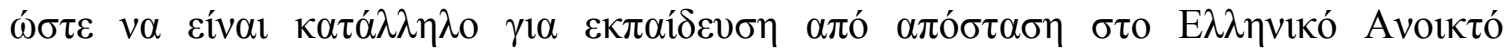

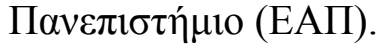

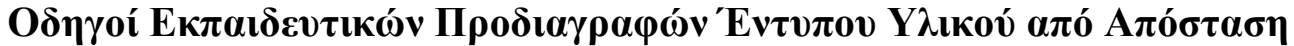

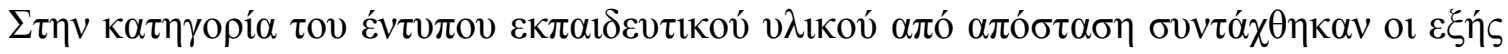

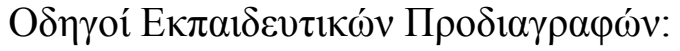

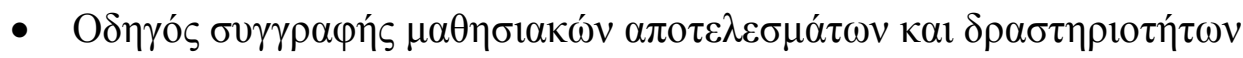

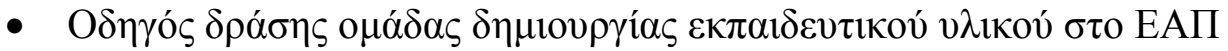

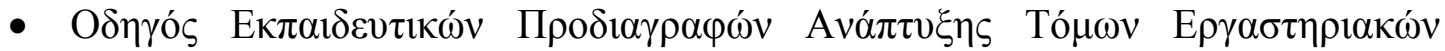
$\Delta \rho \alpha \sigma \tau \eta \rho 10 \tau \eta ́ \tau \omega \nu$

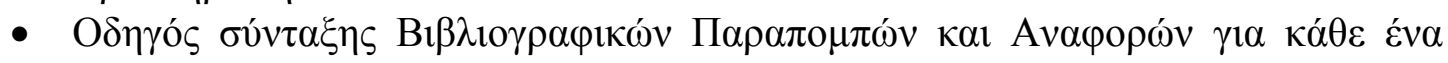

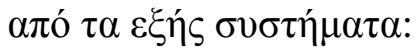
○ APA,
○ Harvard
○ Vancouver
$\circ$ Chicago

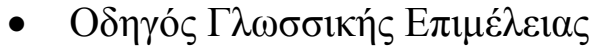

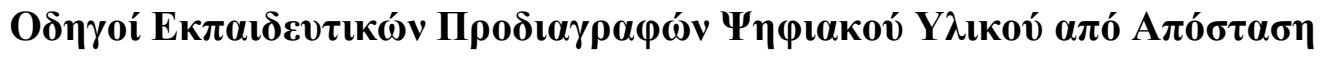

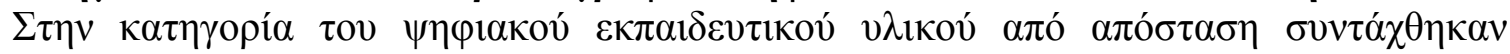

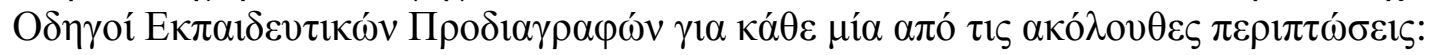

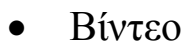

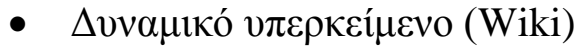

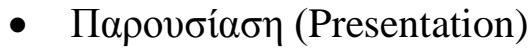

- Y $\pi \varepsilon \rho \mu \varepsilon ́ \sigma o(H y p e r m e d i a)$

- Animation

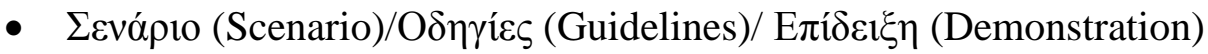

- E

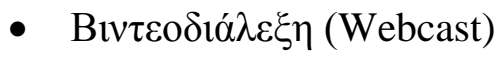

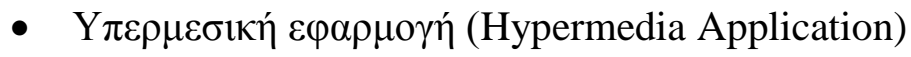

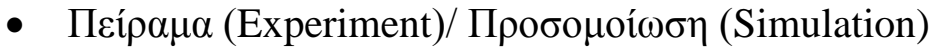

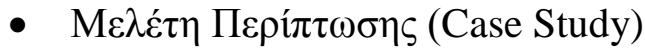

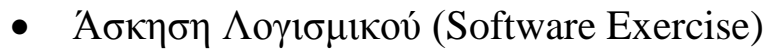

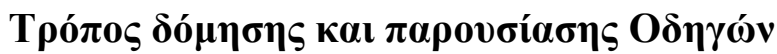

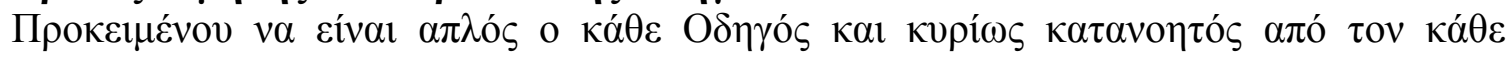

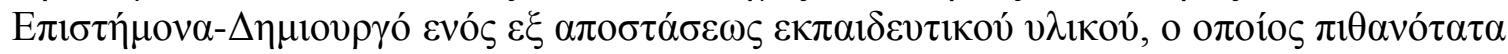

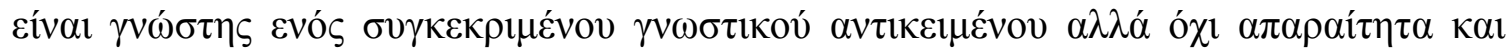

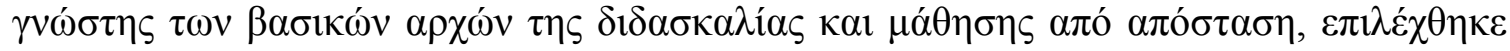

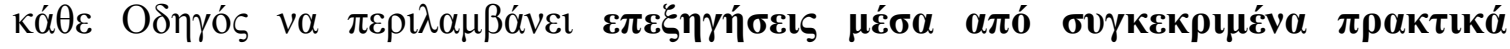

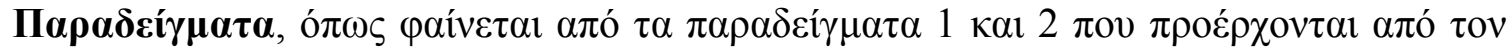

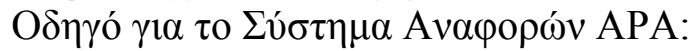




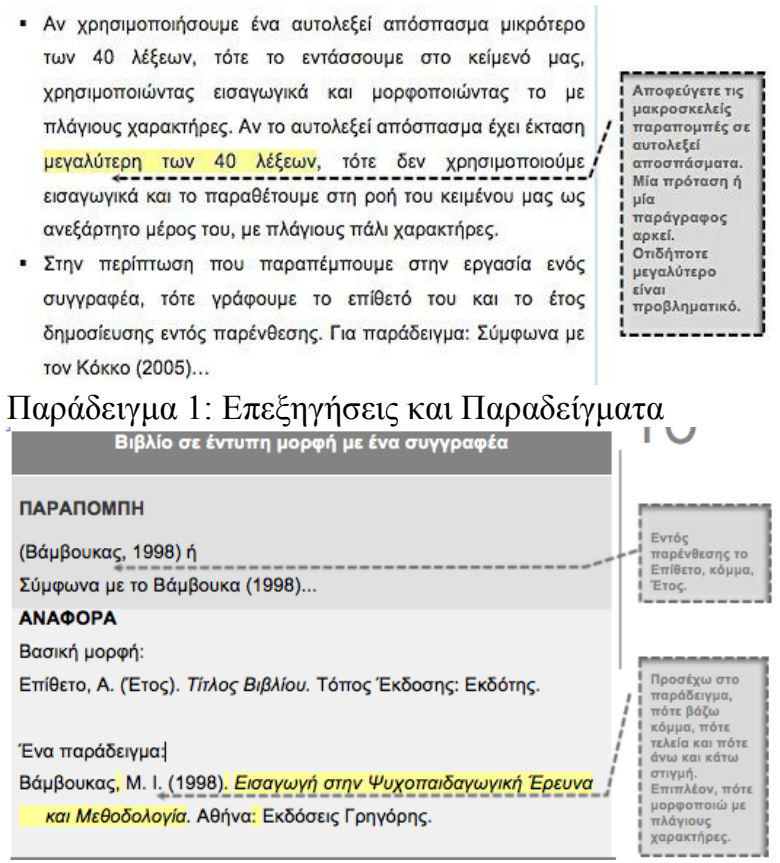

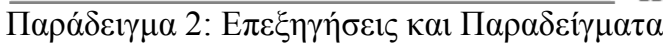

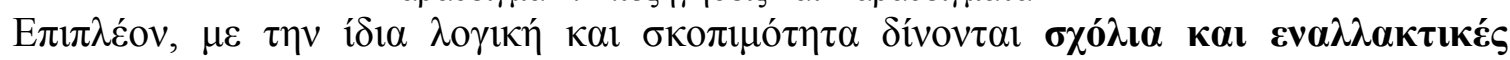

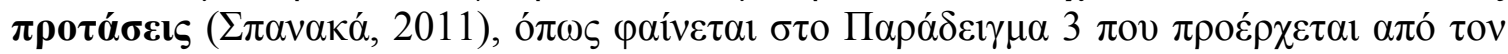

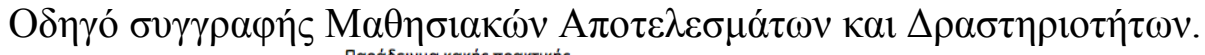

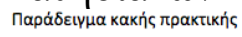

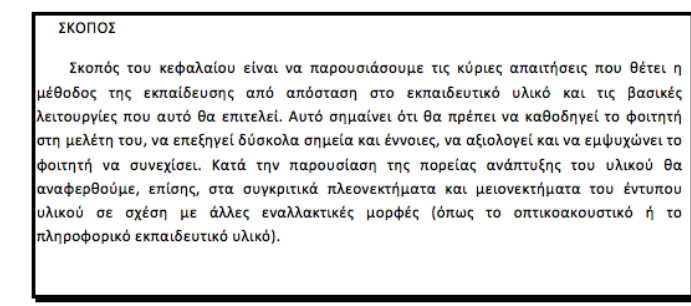

Exó̀ıo

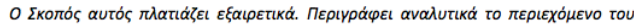

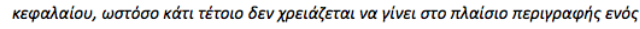

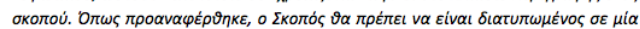

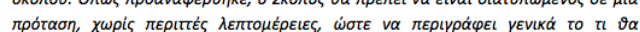

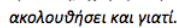

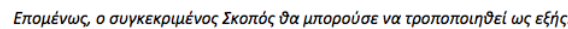

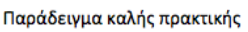

\section{हКопог}

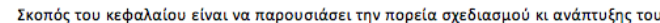

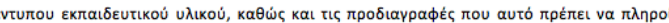

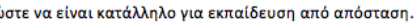

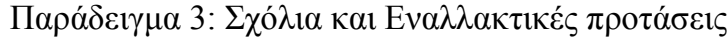

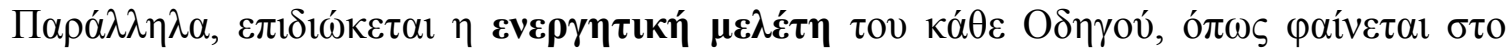

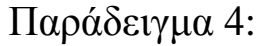

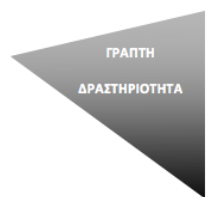

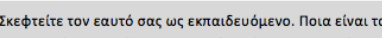

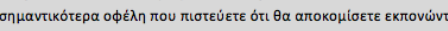

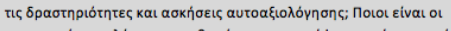

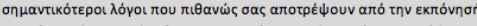

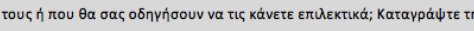

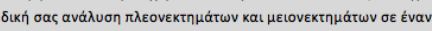

rivaka.

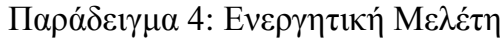

SECTION B: applications, experiences, good practices, descriptions and outlines, educational activities, issues for dialog and discussion 


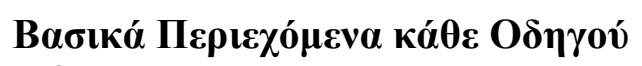

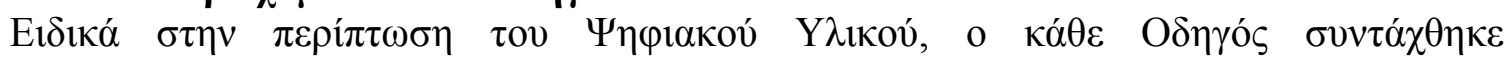

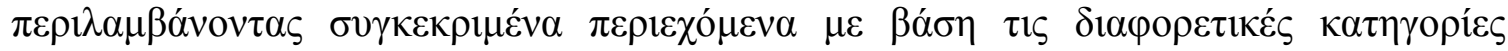

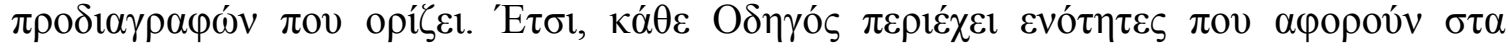
$\alpha \kappa o ́ \lambda o v \theta \alpha \pi \varepsilon \delta i ́ \alpha$ :

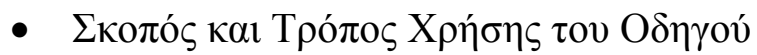

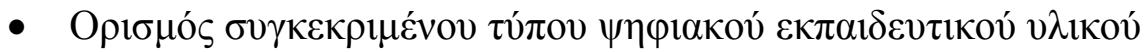

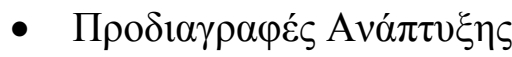

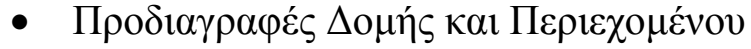

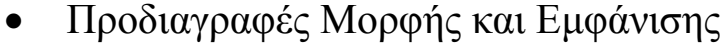

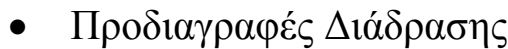

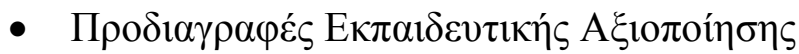

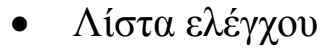

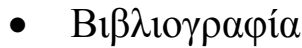

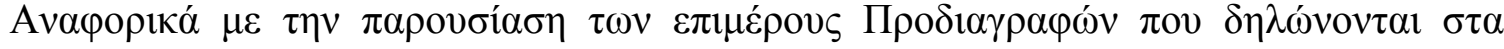

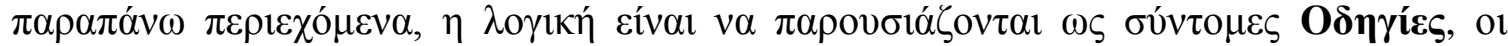

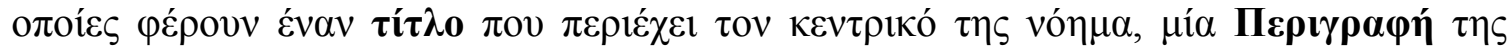

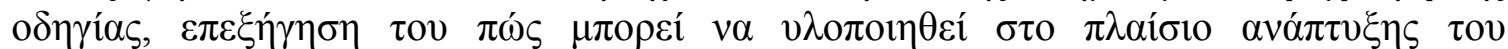

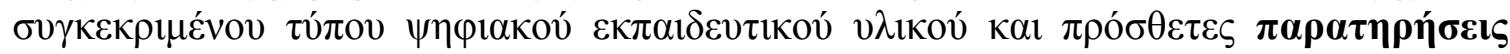

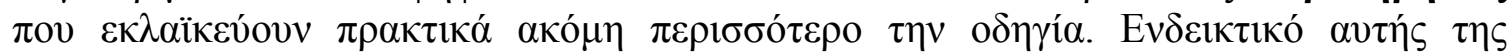

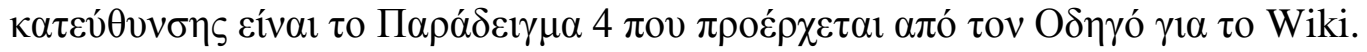

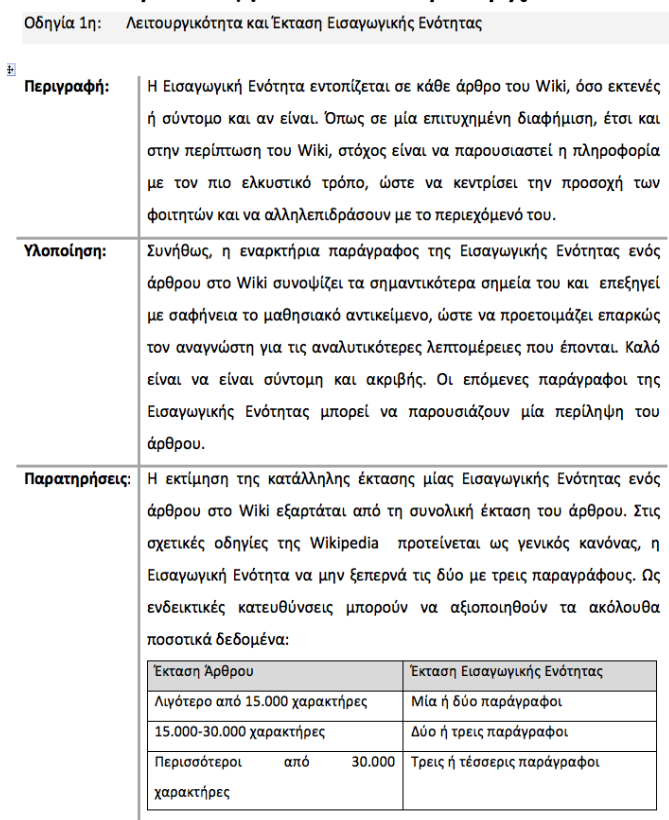

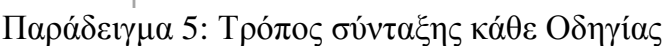

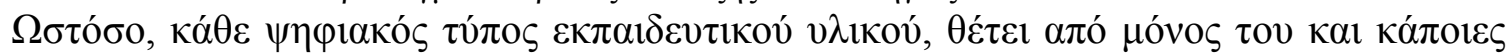

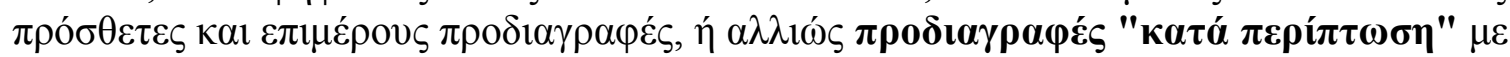

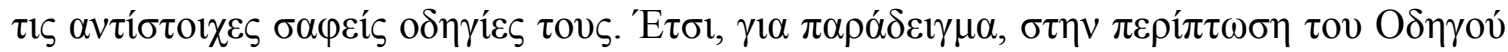

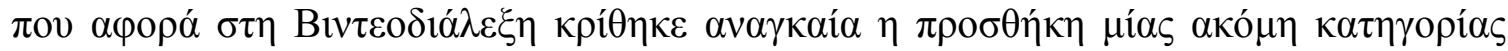

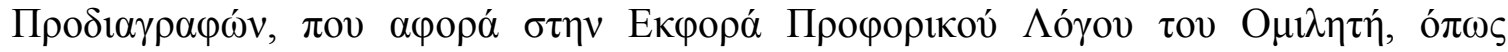

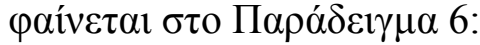




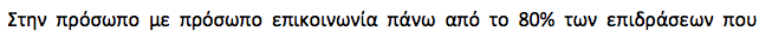

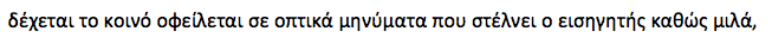

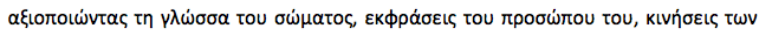

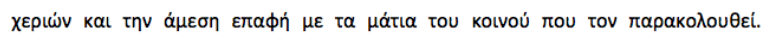

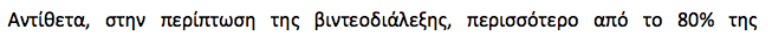

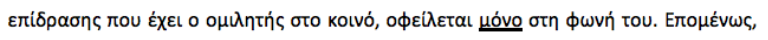

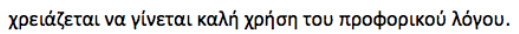

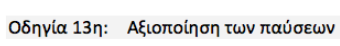

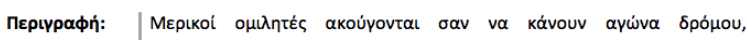

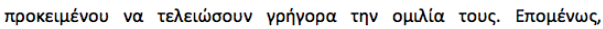

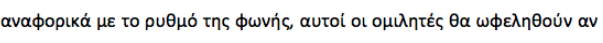

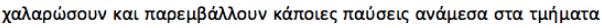

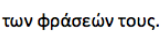

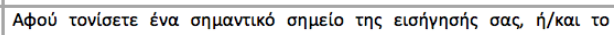

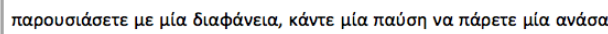

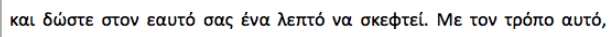

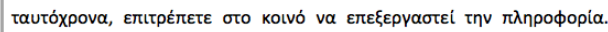

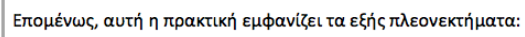

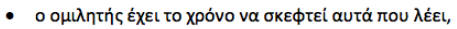

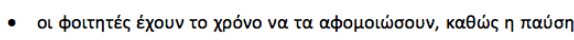

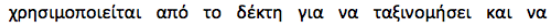

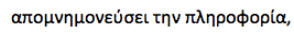

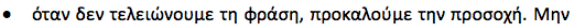

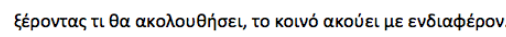

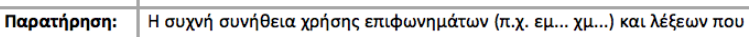

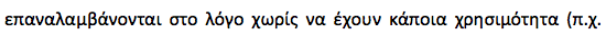

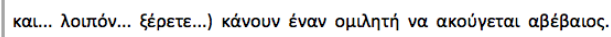

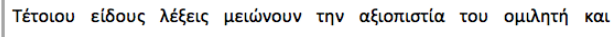

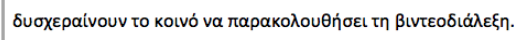

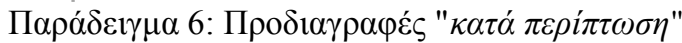

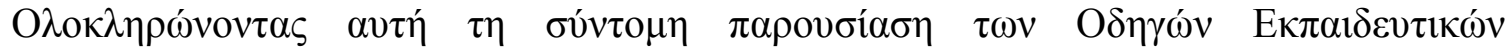

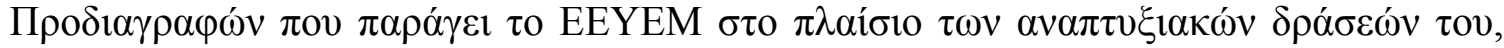

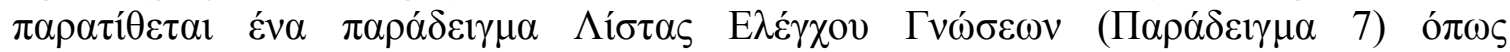

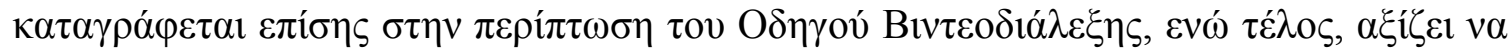

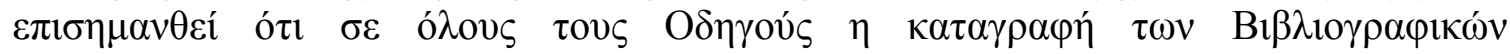

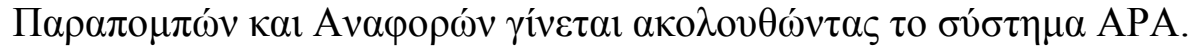

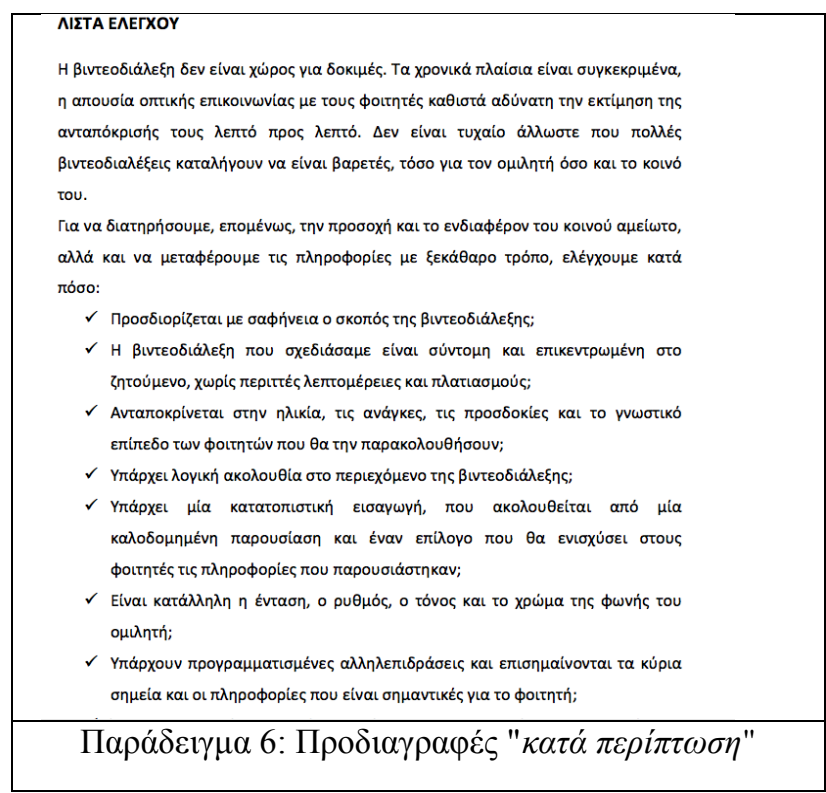

SECTION B: applications, experiences, good practices, descriptions and outlines, educational activities, issues for dialog and discussion 
$7^{\text {th }}$ International Conference in Open \& Distance Learning - November 2013, Athens, Greece - PROCEEDINGS

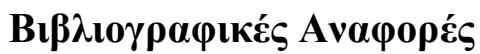

Johnson, E.B. (2002). Contextual teaching and learning: What it is and why it's here to stay. Thousand Oaks, CA: Sage

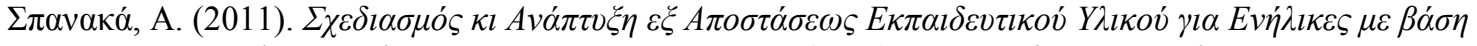

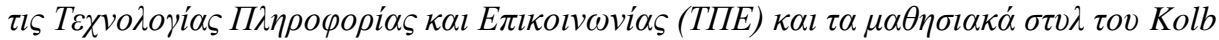

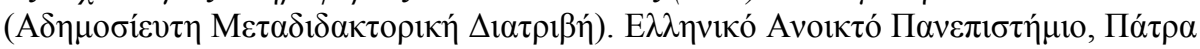

